Short Communication

Correspondence

Radhakrishnan Padmanabhan rp55@georgetown.edu

Received 3 November 2005 Accepted 1 March 2006

\section{Inhibition of dengue virus replication by mycophenolic acid and ribavirin}

\author{
Ratree Takhampunya, ${ }^{1,2}$ Sukathida Ubol, ${ }^{2}$ Huo-Shu Houng, ${ }^{3}$ \\ Craig E. Cameron ${ }^{4}$ and Radhakrishnan Padmanabhan ${ }^{1}$
}

\author{
${ }^{1}$ Department of Microbiology and Immunology, Georgetown University School of Medicine, \\ 3900 Reservoir Road, Washington, DC 20057, USA \\ 2Department of Microbiology, Faculty of Science, Mahidol University, 272 Rama VI Road, \\ Bangkok 10400, Thailand \\ ${ }^{3}$ Department of Virus Diseases, Walter Reed Army Institute of Research, Silver Spring, \\ MD 20910, USA \\ ${ }^{4}$ Department of Biochemistry and Molecular Biology, Pennsylvania State University, University
Park, PA 16802, USA
}

Dengue viruses (DEN), mosquito-borne members of the family Flaviviridae, are human pathogens of global significance. The effects of mycophenolic acid (MPA) and ribavirin (RBV) on DEN replication in monkey kidney (LLC-MK2) cells were examined. MPA $\left(\mathrm{IC}_{50}=0.4 \pm 0.3 \mu \mathrm{M}\right)$ and RBV $\left(\mathrm{IC}_{50}=50 \cdot 9 \pm 18 \mu \mathrm{M}\right)$ inhibited DEN2 replication. Quantitative real-time RT-PCR of viral RNA and plaque assays of virions from DEN2-infected and MPA $(10 \mu \mathrm{M})$ - and RBV $(\geqslant 200 \mu \mathrm{M})$-treated cells showed a fivefold increase in defective viral RNA production by cells treated with each drug. Moreover, a dramatic reduction of intracellular viral replicase activity was seen by in vitro replicase assays. Guanosine reversed the inhibition of these compounds, suggesting that one mode of antiviral action of MPA and RBV is by inhibition of inosine monophosphate dehydrogenase and thereby depletion of the intracellular GTP pool. In addition, RBV may act by competing with guanine-nucleotide precursors in viral RNA translation, replication and 5' capping.
Dengue viruses (DEN1-4), mosquito-borne members of the family Flaviviridae, are human pathogens of global significance. Of the 1 million annual cases of dengue haemorrhagic fever/dengue shock syndrome, about 2-5\% are fatal. Currently, there is no vaccine or antiviral drug to treat DEN infections (Barrett, 2001; Gubler, 1998; Halstead \& Deen, 2002). DEN2 New Guinea C strain, used in this study, has a single-stranded RNA genome (10723 nt) of positive polarity (Irie et al., 1989). The aim of this study was to examine the antiviral action of mycophenolic acid (MPA) and ribavirin (RBV) on DEN2-infected LLC-MK2 cells by determining the number of infectious particles, levels of virion-associated RNAs and intracellular viral replicase activity by plaque assays, quantitative real-time RT-PCR (qRT-PCR) and in vitro replicase assays, respectively. MPA, a non-nucleoside analogue, is a potent, non-competitive inhibitor of inosine monophosphate dehydrogenase (IMPDH), a key enzyme required for biosynthesis of guanine nucleotides (reviewed by Allison \& Eugui, 2000). GTP is required for translation, transcription and replication processes. Therefore, inhibition of IMPDH is expected to inhibit not only proliferation of eukaryotic cells, but also replication of DNA and RNA viruses (Markland et al., 2000). However, RBV, a nucleoside analogue, is a competitive inhibitor of IMPDH.
It is approved as an inhaled drug for treatment of respiratory syncytial virus infection, as well as orally, together with alpha interferon, for treatment of hepatitis $\mathrm{C}$ virus (HCV) infections.

DEN2 was propagated in mosquito (C6/36) cells as described previously (Charnsilpa et al., 2005). LLC-MK2 cells were infected with DEN2 under single-step growth conditions (Dulbecco \& Vogt, 1954) at an m.o.i. of 10 and incubated for $72 \mathrm{~h}$ with $1 \%$ fetal bovine serum. The plaque assay was performed essentially as described previously (Charnsilpa et al., 2005).

To quantify the virus-associated RNA, qRT-PCR was used as described previously (Houng et al., 2000). The detection of PCR product was correlated with input cDNA copy number at various concentrations of antiviral compounds and the results were plotted. DEN2 copy-number standards (ten serial $1: 3$ dilutions of virus stock at $4 \cdot 20 \times 10^{6}$ p.f.u. $\mathrm{ml}^{-1}$ ) were from Walter Reed Army Institute of Research (Washington, DC, USA).

Fig. 1 shows the effect of MPA (Fig. 1a) and RBV (Fig. 1b) at various concentrations on the infectivity of DEN2 and viral RNA copy numbers. The results indicated that, at $5 \mu \mathrm{M}$ 
(a)

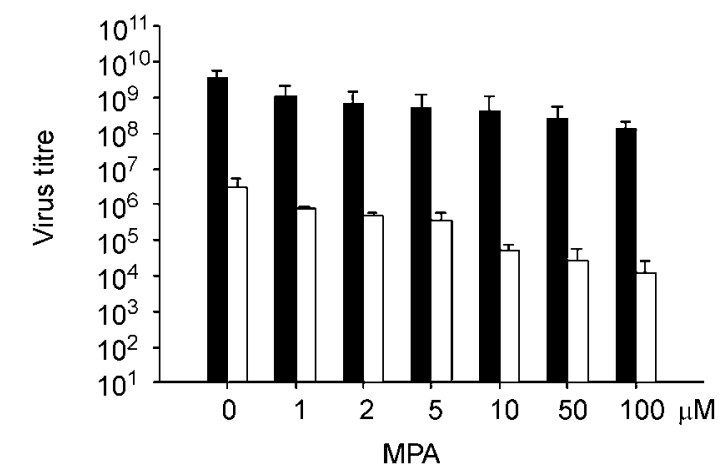

(b)

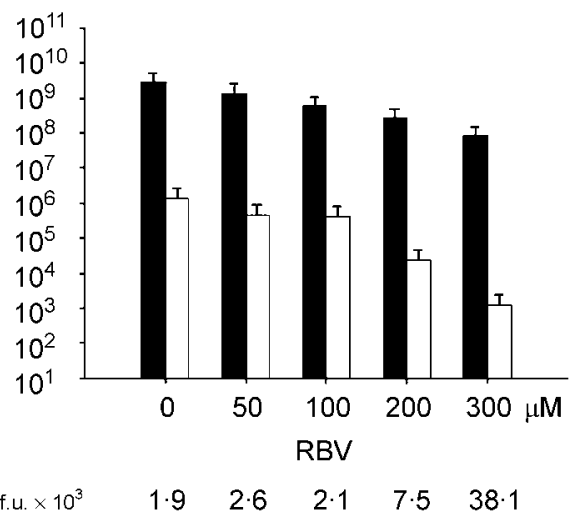

Fig. 1. Inhibition of DEN2 infectivity and viral RNA synthesis by MPA (a) and RBV (b). Aliquots (140 $\mu$ ) of the culture medium, after clarification by centrifugation to remove cellular materials, were used for plaque assays (empty bars; p.f.u. $\mathrm{ml}^{-1}$ ) and to determine vRNA copy number by qRT-PCR (filled bars; RNA copy no. $\mathrm{ml}^{-1}$ ). This experiment was repeated three times with similar results. The ratios of vRNA copy number:p.f.u. $\left(\times 10^{3}\right)$ for the control and various concentrations of MPA and RBV $(\mu \mathrm{M})$ are shown.

MPA, there was a reduction to $91 \pm 7 \cdot 5 \%$ of the untreatedcontrol viral titre, whereas RBV (clinical grade) was required at $150 \mu \mathrm{M}$ for a comparable reduction. The $\mathrm{IC}_{50}$ values of MPA or RBV, calculated from three independent experiments similar to those shown in Fig. 1, were about $0 \cdot 4 \pm 0 \cdot 3$ and $50 \cdot 9 \pm 18 \mu \mathrm{M}$, respectively, based on the median-effect plot (Chou \& Talaly, 1977; Chou et al., 1994).

A previous study reported $\mathrm{EC}_{50}$ values of $0 \cdot 3 \pm 0 \cdot 2$ and $155 \cdot 6 \pm 45 \mu \mathrm{M}$ for MPA and RBV, respectively, for inhibition of DEN replication in Vero cells (Leyssen et al., 2001). Although these values for MPA in LLC-MK2 and Vero cells are very similar, there is about a threefold difference in the potency of RBV $(50 \cdot 9 \pm 18$ versus $155 \cdot 6 \pm 45 \mu \mathrm{M})$. This variance might be related to the differences in the batches of RBV used or due to intrinsic differences in the drug sensitivities of the two cell lines. The $\mathrm{IC}_{50}$ values of MPA for inhibition of DEN2 infectivity in four human hepatoma cell lines (Hep3B, HepG2, CRL-8024 and Huh-7) were 0·3, $3,1 \cdot 2$ and $1.9 \mu \mathrm{M}$ and those of RBV were $20,60,100$ and $40 \mu \mathrm{M}$, respectively (Diamond et al., 2002). Thus, the antiviral potencies of MPA and RBV varied, depending on the cell type used and growth properties (human versus primate or normal versus transformed). This conclusion is supported by recent studies. DEN replicated better in cycling cells than in density-arrested mosquito (C6/36) cells and this difference was not seen in human hepatoma cells (Helt \& Harris, 2005). Moreover, West Nile virus replication was abrogated after four passages in HeLa cells in the presence of RBV, whereas high titres persisted even after many passages in other cell lines, such as Vero or CV1. Differences in phosphorylation of RBV between different cell types may also account for differences in its antiviral activity (Smee et al., 2001). RBV is converted to the mono(RMP)-, di(RDP)- and tri(RTP)-phosphorylated forms within eukaryotic cells. For example, a 13-fold more efficient phosphorylation of RBV in the mouse 3T3 cell line in contrast to Vero cells may account for differences in the efficacy of RBV against West Nile virus infection of Vero cells (Morrey et al., 2002) or BHK-21 cells (Lo et al., 2003). In the latter study, a West Nile virus replicon RNA expressing the Renilla luciferase reporter was used and the $\mathrm{EC}_{50}$ values of MPA and RBV were $5 \cdot 4$ and $140 \mu \mathrm{M}$, respectively.

Moreover, our study showed that the infectivity of the virus released into the medium was more sensitive to MPA or RBV inhibition than the viral RNA copy numbers, as shown by the ratio of vRNA : p.f.u. for each concentration of MPA and RBV. The data indicated that, at MPA concentrations of $1-5 \mu \mathrm{M}$, the ratios of vRNA:p.f.u. were around 2000, whereas at higher concentrations of MPA $(10-100 \mu \mathrm{M})$, the ratio increased to approximately 11000 and, in the untreated control, the ratio remained at approximately 1115 (Fig. 1a). The results shown in Fig. 1(b) indicated that the virus titre and the vRNA copy number in the culture supernatants decreased with increasing RBV concentrations. The vRNA:p.f.u. ratio remained without significant change up to $100 \mu \mathrm{M} \mathrm{RBV}$, but, at 200 and $300 \mu \mathrm{M}$, it followed a trend similar to that of MPA. These results showed that MPA at $10 \mu \mathrm{M}$ and RBV at $\geqslant 200 \mu \mathrm{M}$ affected the infectivity of virions released into the culture medium significantly.

The effects of MPA and RBV on cell viability were analysed by using a TOX-1 kit (Sigma). This assay is based on the activity of mitochondrial dehydrogenases of living cells, which convert a chromogenic substrate [3-(4,5-dimethylthiazol-2-yl)-2,5-diphenyltetrazolium bromide; MTT] into purple formazan. It was quantified by measuring $A_{570}$ using a spectrophotometer according to the manufacturer's protocol. Cells were viable in the presence of MPA and RBV up to 100 and $300 \mu \mathrm{M}$, respectively, at $90-100 \%$ of untreatedcontrol cells (data not shown). 
MPA and RBV as RMP are inhibitors of IMPDH in vitro (Streeter et al., 1973) and in vivo (Muller et al., 1977). Hence, one possible mode of inhibition of virus replication by MPA and RBV is by depletion of the intracellular pool of guanosine nucleotides, thereby inhibiting viral RNA synthesis. In addition, RBV as RTP may inhibit by competing with GTP required for translation, replication and 5' capping of viral RNA. To test this possibility, different amounts of guanosine were added to the culture medium containing a fixed concentration of MPA $(50 \mu \mathrm{M})$, a concentration at which the viral titre was reduced significantly by at least two orders of magnitude (Fig. 1). However, additions of 250 or $500 \mu \mathrm{M}$ or $1 \cdot 0 \mathrm{mM}$ guanosine (five-, 10 and 20-fold excess over MPA, respectively) restored infectivity nearly to the untreated-control levels (Fig. 2a). Similar reversal of RBV inhibition was also seen by guanosine (Fig. 2b). These results suggest that one mechanism of antiviral activity of MPA and RBV is through inhibition of IMPDH, thereby depleting the intracellular guaninenucleotide pool and causing misincorporation of nucleotides by the error-prone RNA-dependent RNA polymerase (RdRP) to produce defective genomes. This mechanism of action was also suggested in an earlier study (Diamond et al., 2002). In addition, RBV may also act in part by competition with GTP. The finding that inhibitory effects of MPA and RBV are reversible by exogenous addition of guanosine supports this conclusion.

NS5 is the RdRP of mosquito-borne flaviviruses and its activity in RNA synthesis was demonstrated as a component of crude, membrane-bound viral replicase complexes from flavivirus-infected cells (Chu \& Westaway, 1987; Grun \& Brinton, 1986; Uchil \& Satchidanandam, 2003; You \& Padmanabhan, 1999) or as a purified protein (Ackermann \& Padmanabhan, 2001; Guyatt et al., 2001; Tan et al., 1996). The N-terminal domain of NS5 has a 2'-O-methyltransferase activity (Egloff et al., 2002) and RTP inhibits this activity (Benarroch et al., 2004). An in vitro viral replicase assay based on endogenous membrane-bound viral RNA, associated with viral replicase, is pivotal to our current understanding of flavivirus replication (Chu \& Westaway, 1985; Uchil \& Satchidanandam, 2003; reviewed by Westaway et al., 2002). Three intracellular species of RNA are produced during flavivirus replication in mammalian cells that are separable by electrophoresis on a partially denaturing polyacrylamide/7 $\mathrm{M}$ urea gel: a $44 \mathrm{~S}$ genome length single-stranded viral RNA (vRNA), 20S doublestranded replicative form (RF) and the 20-28S partially nuclease-resistant replicative intermediate (RI) (Chu \& Westaway, 1985). According to the current model, viral RNA is converted first to RF; RF serves as a recycling template for semiconservative and essentially asymmetric replication to produce one progeny single strand per cycle (Westaway et al., 2003).

Next, we examined whether MPA and RBV affect the activity of the viral replicase to produce the three intracellular RNA species in vitro. Membrane-associated viral replicase complexes $(20 \mu \mathrm{g}$ total protein) were prepared from DEN2infected LLC-MK2 cells treated with MPA or RBV at various concentrations for $48 \mathrm{~h}$. In vitro RNA synthesis was initiated by addition of the four NTPs containing $\alpha{ }^{32} \mathrm{P}$-labelled GTP and the ATP-regenerating system. After incubation, the labelled RNA species were analysed by partially denaturing PAGE. As shown in Fig. 3(a), RNA synthesis in DEN2infected and MPA-treated cells was reduced at concentrations of MPA as low as $0 \cdot 1 \mu \mathrm{M}$ (lane 2) compared with the untreated control (lane 1) and further reduced significantly by MPA at 1 and $2 \mu \mathrm{M}$ (Fig. 3a, lanes 3 and 4). Similarly, treatment with RBV at 50,100 and $200 \mu \mathrm{M}$ reduced the replicative RNA species significantly (Fig. 3b, lanes 2-4). These results suggest that the levels and/or the assembly of a functional viral replicase on the membranes were affected severely by treatment with MPA or RBV at their (a)

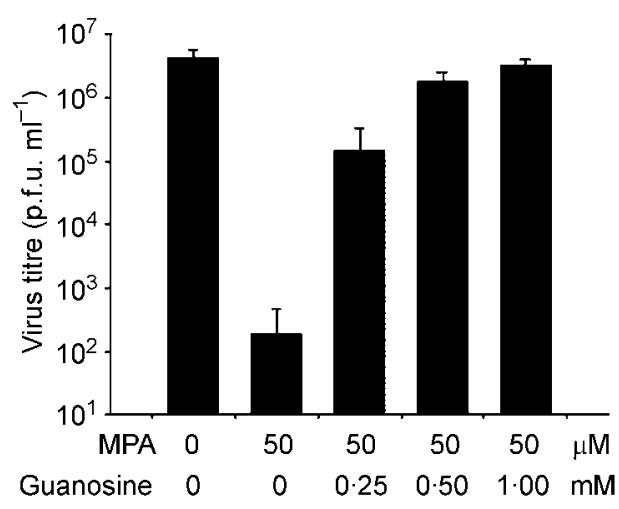

(b)

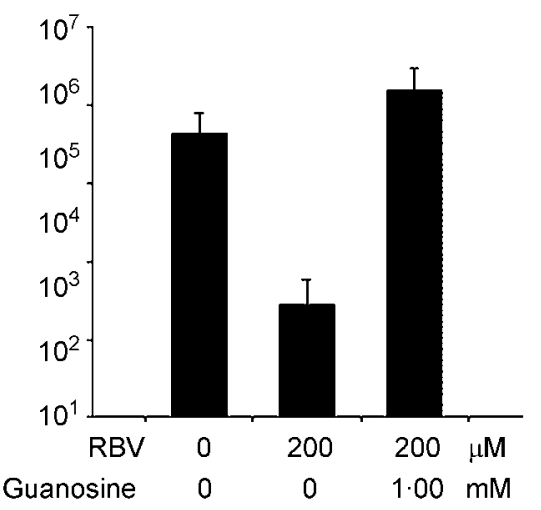

Fig. 2. Reversal of MPA and RBV inhibition of DEN2 replication by exogenous addition of guanosine. DEN2 infection of LLC-MK2 cells and treatment with MPA $(50 \mu \mathrm{M})$ or RBV $(200 \mu \mathrm{M})$ in the presence or absence of guanosine at indicated concentrations are shown. After $72 \mathrm{~h}$, the media were collected and virus titres (p.f.u. $\mathrm{ml}^{-1}$ ) were determined as described in the text. Data from three and two independent experiments in (a) and (b), respectively, were plotted. 

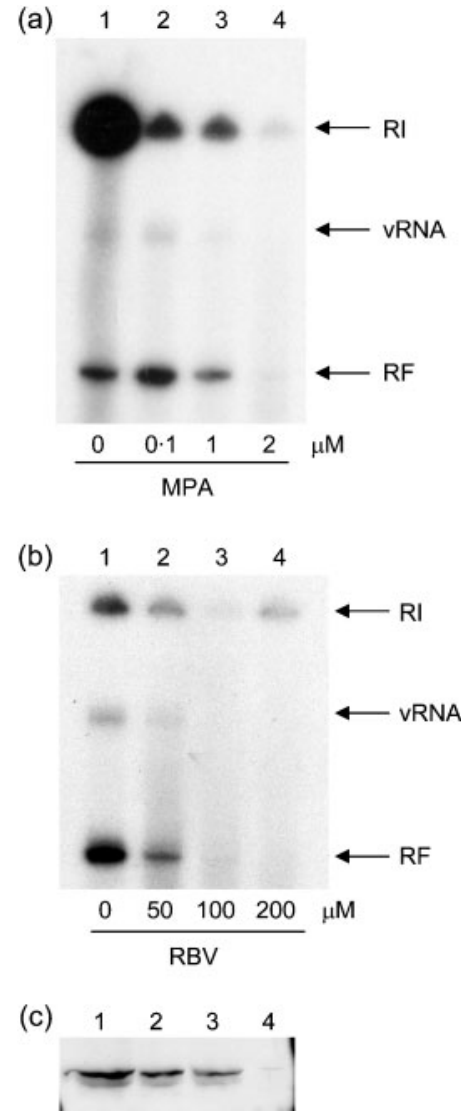

Fig. 3. In vitro viral replicase assays and intracellular levels of NS5. LLC-MK2 cells were infected with DEN2 and treated with MPA (a) or RBV (b) at indicated concentrations. After $48 \mathrm{~h}$ incubation, cell lysates were prepared and aliquots of total protein $(20 \mu \mathrm{g})$ were used for in vitro viral replicase assays. The products were analysed by polyacrylamide (3\%)/7 M urea gels and autoradiography. The autoradiograph is representative of two independent experiments. (c) MPA-treated cell lysates were subjected to Western blotting using rabbit polyclonal anti-NS5 antibodies.

corresponding $\mathrm{IC}_{50}$ concentrations. Moreover, MPA at $10 \mu \mathrm{M}$ or RTP up to $1 \mathrm{mM}$ did not inhibit RNA synthesis to a detectable extent when added directly to the in vitro viral replicase assays. However, 100-fold less MPA and tenfold less RBV than the nucleoside had a dramatic effect of inhibiting virus replication in the infected cell cultures (Fig. 3b), indicating that the inhibitory effect of MPA and RBV on viral RNA synthesis was indirect (data not shown). Thus, the results of the in vitro assays using endogenous viral replicase (Chu \& Westaway, 1987) from the antiviral compoundtreated cells confirm the inhibitory effects of MPA and RBV, as seen by infectivity assays and RNA copy number estimation by qRT-PCR.

If MPA reduces the intracellular pool of GTP and thereby inhibits viral RNA synthesis and the release of infectious virions, it would eventually be expected to reduce the number of viral RNA templates available for translation followed by polyprotein processing, the assembly of the replicase complex and the levels of NS5. To test this possibility, lysates from DEN2-infected cells, either untreated or treated with MPA at $0 \cdot 1,1$ and $2 \mu \mathrm{M}$ concentrations, were analysed by Western blotting with polyclonal rabbit antiNS5 antibodies (1:100; M. Ackermann \& R. Padmanabhan, unpublished data) and horseradish peroxidase-labelled goat anti-rabbit antibody $(1: 2000)$. NS5 was detected by chemiluminescence (ECL system; Amersham Biosciences). The results shown in Fig. 3(c) indicate that the level of intracellular NS5 was reduced significantly upon treatment with MPA at $0 \cdot 1$ and $1 \mu \mathrm{M}$ and was barely detectable at $2 \mu \mathrm{M}$.

For a drug to be of therapeutic value, the dosage of the drug should be adjusted so that it has maximum inhibitory effect with lowest cytotoxicity to the host. The immunosuppressive drug mycophenolate mofetil is given as part of a drug regimen to organ-transplant recipients; concentrations of $1 \cdot 5-3 \cdot 0 \mu \mathrm{g} \mathrm{ml}^{-1}$ (approx. 3.5-6.5 $\mu \mathrm{M}$ ) are attained easily in human plasma upon oral dosing (Bullingham et al., 1996).

Our results that a fivefold increase in the ratio of vRNA:p.f.u. occurs at MPA $\geqslant 10 \mu \mathrm{M}$ or $\mathrm{RBV} \geqslant 200 \mu \mathrm{M}$ could be explained by generation of defective quasispecies of viral RNA when the GMP pool is progressively depleted and/or outcompeted by phosphorylated forms of RBV during replication of viral RNA by the error-prone RdRP. In fact, treatment of cells with RBV caused a twofold reduction in intracellular GTP levels (Muller et al., 1977). This conclusion is also consistent with our results that antiviral activity of MPA and RBV was reversed by the addition of excess guanosine. A recent study also concluded that the inhibition of IMPDH by RBV is the major mechanism for its antiviral activity (Leyssen et al., 2005).

Evidence from other studies also indicated that the antiviral activity of RBV could be explained by an alternative mechanism. Poliovirus and HCV RdRPs could utilize the RTP precursor and incorporate RMP into RNA complementary to either cytidine or uridine in a template-directed primer-extension assay. These results suggested that RMP incorporation into viral RNA would be expected to produce mutant genomes during replication (Crotty et al., 2000, 2001; Maag et al., 2001; reviewed by Graci \& Cameron, 2002). In another study using a short, synthetic RNA template (LE19), HCV NS5B polymerase, UTP, CTP and RTP, RMP was incorporated, albeit inefficiently, into a primerextension product. Moreover, accumulation of transition mutations was revealed by sequencing the West Nile virus RNA synthesized in the presence of RBV, suggesting that RBV induced error-prone replication (Day et al., 2005).

\section{Acknowledgements}

This study constitutes partial fulfilment for the degree of Doctor of Philosophy for R.T. from the Department of Microbiology and Immunology, Mahidol University, Bangkok, Thailand. The work was done at Georgetown University under the mentorship of R.P. This 
work was supported by NIH grants R01 AI-32078 (R.P.) and U01 AI 54776 (C. E. C. and R. P.), a predoctoral fellowship from the Ministry of Education (026/2544), Thailand, and a Thailand Research Fund grant (BRG 4580019). We thank Jason Graci for critical reading of the manuscript.

\section{References}

Ackermann, M. \& Padmanabhan, R. (2001). De novo synthesis of RNA by the dengue virus RNA-dependent RNA polymerase exhibits temperature dependence at the initiation but not elongation phase. J Biol Chem 276, 39926-39937.

Allison, A. C. \& Eugui, E. M. (2000). Mycophenolate mofetil and its mechanisms of action. Immunopharmacology 47, 85-118.

Barrett, A. D. T. (2001). Current status of flavivirus vaccines. Ann N Y Acad Sci 951, 262-271.

Benarroch, D., Egloff, M.-P., Mulard, L., Guerreiro, C., Romette, J.-L. \& Canard, B. (2004). A structural basis for the inhibition of the NS5 dengue virus mRNA 2'-O-methyltransferase domain by ribavirin 5'-triphosphate. J Biol Chem 279, 35638-35643.

Bullingham, R., Monroe, S., Nicholls, A. \& Hale, M. (1996). Pharmacokinetics and bioavailability of mycophenolate mofetil in healthy subjects after single-dose oral and intravenous administration. J Clin Pharmacol 36, 315-324.

Charnsilpa, W., Takhampunya, R., Endy, T. P., Mammen, M. P., Jr, Libraty, D. H. \& Ubol, S. (2005). Nitric oxide radical suppresses replication of wild-type dengue 2 viruses in vitro. J Med Virol 77, 89-95.

Chou, T.-C. \& Talaly, P. (1977). A simple generalized equation for the analysis of multiple inhibitions of Michaelis-Menten kinetic systems. $J$ Biol Chem 252, 6438-6442.

Chou, T.-C., Motzer, R. J., Tong, Y. \& Bosl, G. J. (1994). Computerized quantitation of synergism and antagonism of taxol, topotecan, and cisplatin against human teratocarcinoma cell growth: a rational approach to clinical protocol design. J Natl Cancer Inst 86, $1517-1524$.

Chu, P. W. G. \& Westaway, E. G. (1985). Replication strategy of Kunjin virus: evidence for recycling role of replicative form RNA as template in semiconservative and asymmetric replication. Virology 140, 68-79.

Chu, P. W. G. \& Westaway, E. G. (1987). Characterization of Kunjin virus RNA-dependent RNA polymerase: reinitiation of synthesis in vitro. Virology 157, 330-337.

Crotty, S., Maag, D., Arnold, J. J., Zhong, W., Lau, J. Y. N., Hong, Z., Andino, R. \& Cameron, C. E. (2000). The broad-spectrum antiviral ribonucleoside ribavirin is an RNA virus mutagen. Nat Med 6, 1375-1379.

Crotty, S., Cameron, C. E. \& Andino, R. (2001). RNA virus error catastrophe: direct molecular test by using ribavirin. Proc Natl Acad Sci U S A 98, 6895-6900.

Day, C. W., Smee, D. F., Julander, J. G., Yamshchikov, V. F., Sidwell, R. W. \& Morrey, J. D. (2005). Error-prone replication of West Nile virus caused by ribavirin. Antiviral Res 67, 38-45.

Diamond, M. S., Zachariah, M. \& Harris, E. (2002). Mycophenolic acid inhibits dengue virus infection by preventing replication of viral RNA. Virology 304, 211-221.

Dulbecco, R. \& Vogt, M. (1954). One-step growth curve of Western equine encephalomyelitis virus on chicken embryo cells grown in vitro and analysis of virus yields from single cells. J Exp Med 99, 183-199.

Egloff, M.-P., Benarroch, D., Selisko, B., Romette, J.-L. \& Canard, B. (2002). An RNA cap (nucleoside-2'-O-)-methyltransferase in the flavivirus RNA polymerase NS5: crystal structure and functional characterization. EMBO J 21, 2757-2768.

Graci, J. D. \& Cameron, C. E. (2002). Quasispecies, error catastrophe, and the antiviral activity of ribavirin. Virology 298, 175-180.

Grun, J. B. \& Brinton, M. A. (1986). Characterization of West Nile virus RNA-dependent RNA polymerase and cellular terminal adenylyl and uridylyl transferases in cell-free extracts. J Virol 60, 1113-1124.

Gubler, D. J. (1998). Dengue and dengue hemorrhagic fever. Clin Microbiol Rev 11, 480-496.

Guyatt, K. J., Westaway, E. G. \& Khromykh, A. A. (2001). Expression and purification of enzymatically active recombinant RNA-dependent RNA polymerase (NS5) of the flavivirus Kunjin. J Virol Methods 92, 37-44.

Halstead, S. B. \& Deen, J. (2002). The future of dengue vaccines. Lancet 360, 1243-1245.

Helt, A.-M. \& Harris, E. (2005). S-phase-dependent enhancement of dengue virus 2 replication in mosquito cells, but not in human cells. J Virol 79, 13218-13230.

Houng, H.-S. H., Hritz, D. \& Kanesa-Thasan, N. (2000). Quantitative detection of dengue 2 virus using fluorogenic RT-PCR based on 3'-noncoding sequence. J Virol Methods 86, 1-11.

Irie, K., Mohan, P. M., Sasaguri, Y., Putnak, R. \& Padmanabhan, R. (1989). Sequence analysis of cloned dengue virus type 2 genome (New Guinea-C strain). Gene 75, 197-211.

Leyssen, P., Van Lommel, A., Drosten, C., Schmitz, H., De Clercq, E. \& Neyts, J. (2001). A novel model for the study of the therapy of flavivirus infections using the Modoc virus. Virology 279, 27-37.

Leyssen, P., Balzarini, J., De Clercq, E. \& Neyts, J. (2005). The predominant mechanism by which ribavirin exerts its antiviral activity in vitro against flaviviruses and paramyxoviruses is mediated by inhibition of IMP dehydrogenase. J Virol 79, 1943-1947.

Lo, M. K., Tilgner, M. \& Shi, P.-Y. (2003). Potential high-throughput assay for screening inhibitors of West Nile virus replication. J Virol 77, 12901-12906.

Maag, D., Castro, C., Hong, Z. \& Cameron, C. E. (2001). Hepatitis $\mathrm{C}$ virus RNA-dependent RNA polymerase (NS5B) as a mediator of the antiviral activity of ribavirin. J Biol Chem 276, 46094-46098.

Markland, W., McQuaid, T. J., Jain, J. \& Kwong, A. D. (2000). Broadspectrum antiviral activity of the IMP dehydrogenase inhibitor VX-497: a comparison with ribavirin and demonstration of antiviral additivity with alpha interferon. Antimicrob Agents Chemother 44, 859-866.

Morrey, J. D., Smee, D. F., Sidwell, R. W. \& Tseng, C. (2002). Identification of active antiviral compounds against a New York isolate of West Nile virus. Antiviral Res 55, 107-116.

Muller, W. E., Maidhof, A., Taschner, H. \& Zahn, R. K. (1977). Virazole (1- $\beta$-D-ribofuranosyl-1,2,4-triazole-3-carboxamide): a cytostatic agent. Biochem Pharmacol 26, 1071-1075.

Smee, D. F., Bray, M. \& Huggins, J. W. (2001). Antiviral activity and mode of action studies of ribavirin and mycophenolic acid against orthopoxviruses in vitro. Antivir Chem Chemother 12, 327-335.

Streeter, D. G., Witkowski, J. T., Khare, G. P., Sidwell, R. W., Bauer, R. J., Robins, R. K. \& Simon, L. N. (1973). Mechanism of action of 1- $\beta$-D-ribofuranosyl-1,2,4-triazole-3-carboxamide (Virazole), a new broad-spectrum antiviral agent. Proc Natl Acad Sci U S A 70, 1174-1178.

Tan, B.-H., Fu, J., Sugrue, R. J., Yap, E.-H., Chan, Y.-C. \& Tan, Y. H. (1996). Recombinant dengue type 1 virus NS5 protein expressed in Escherichia coli exhibits RNA-dependent RNA polymerase activity. Virology 216, 317-325. 
Uchil, P. D. \& Satchidanandam, V. (2003). Characterization of RNA synthesis, replication mechanism, and in vitro RNA-dependent RNA polymerase activity of Japanese encephalitis virus. Virology 307, 358-371.

Westaway, E. G., Mackenzie, J. M. \& Khromykh, A. A. (2002). Replication and gene function in Kunjin virus. Curr Top Microbiol Immunol 267, 323-351.
Westaway, E. G., Mackenzie, J. M. \& Khromykh, A. A. (2003). Kunjin RNA replication and applications of Kunjin replicons. Adv Virus Res 59, 99-140.

You, S. \& Padmanabhan, R. (1999). A novel in vitro replication system for Dengue virus: initiation of RNA synthesis at the $3^{\prime}$-end of exogenous viral RNA templates requires $5^{\prime}$ - and $3^{\prime}$-terminal complementary sequence motifs of the viral RNA. J Biol Chem 274, 33714-33722. 